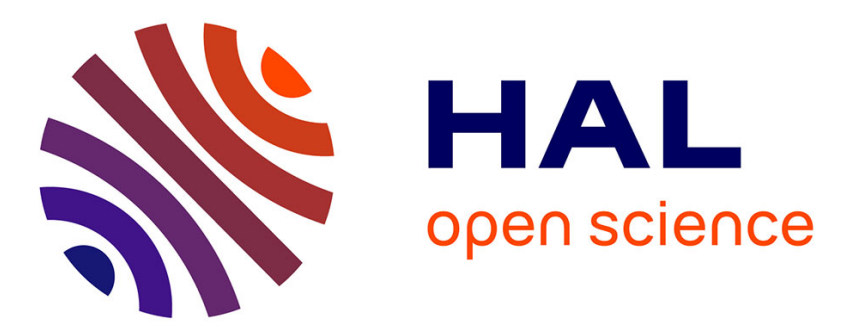

\title{
Wetting and friction on superoleophobic surfaces
}

Laurent Joly, Thierry Biben

\section{To cite this version:}

Laurent Joly, Thierry Biben. Wetting and friction on superoleophobic surfaces. Soft Matter, 2009, 5, pp.2549. 10.1039/b821214g . hal-00437090

\section{HAL Id: hal-00437090 https://hal.science/hal-00437090}

Submitted on 29 Nov 2010

HAL is a multi-disciplinary open access archive for the deposit and dissemination of scientific research documents, whether they are published or not. The documents may come from teaching and research institutions in France or abroad, or from public or private research centers.
L'archive ouverte pluridisciplinaire HAL, est destinée au dépôt et à la diffusion de documents scientifiques de niveau recherche, publiés ou non, émanant des établissements d'enseignement et de recherche français ou étrangers, des laboratoires publics ou privés. 


\title{
Wetting and friction on superoleophobic surfaces
}

\author{
March 20, 2009
}

Laurent Joly and Thierry Biben

Université de Lyon, F-69000, Lyon, France; Université Lyon 1, F-69622, Villeurbanne, France; CNRS, UMR5586, Laboratoire PMCN. E-mail: laurent.joly@univ-lyon1.fr

This article describes the physics of superoleophobic surfaces, combining a recently developed free-energy approach and finite element modeling. We start by investigating the peculiar wetting behavior of such surfaces, originating in their "re-entrant" geometry, and discussing a specific mechanism for the "filling transition", leading to the loss of superoleophobicity. We then focus on their friction properties, and establish an important condition for their use as super-lubricating surfaces. This new insight into the underlying mechanisms of superoleophobicity enables us to suggest general principles to optimize the wetting and friction properties of those promising surfaces.

\section{Introduction}

a) Wenzel state

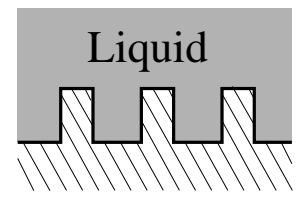

b) Cassie-Baxter state

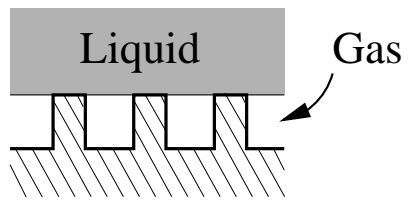

Figure 1: Wenzel and Cassie-Baxter states for patterned hygrophobic surfaces.

Since the late nineties, micro or nano-structured surfaces have attracted a lot of attention in different fields ${ }^{1-11}$. This interest is motivated by the "super-wetting" properties of these surfaces (superhydrophobicity, superhydrophilicity, superoleophobicity, superoleophilicity, ..., generically called superhygrophobicity or superhygrophilicity, as suggested in ${ }^{12}$, whatever the nature of the liquid) and by the consequences these properties have on various transport phenomena at the surface (in particular, matter ${ }^{13}$ and heat ${ }^{10}$ transfert). One important field of application is microfluidic ${ }^{14}$, where superhydrophobicity of suitably prepared textured surfaces can lower in a substantial way the friction between the surface and water, reducing the viscous dissipation in the device ${ }^{13,15-18}$. Similar surfaces can be produced to repel oil, but repelling both oil and water with the same surface is a true challenge since surfaces that repel water are usually attracting oils. The recently introduced "re-entrant" geometries ${ }^{12,19-23}$ (see for instance Fig. 5.b) are interesting in this context since these structures can drive a wetting inversion, leading to surfaces repelling both water and oils (traditionally referred to as superoleophobic surfaces). The geometrical reason responsible for the wetting inversion has been investigated by Marmur in ${ }^{12}$, and results from the multivaluation of the surface (several solutions exist for a given liquid-gas contact area, as defined in ${ }^{12}$ ). As a consequence, a Cassie-Baxter state can exist even when the surface is intrinsically wetting. The surfaces we consider have this property. But this peculiar behavior only appears as a metastable state as we shall see, and controlling the mechanism of metastability is the key point. Investigating these mechanisms is the goal of the present study, that extends in some way the work of Marmur. Another important field of application concerns thermal transport, where superhygrophilicity of some nanostructured surfaces can be used to increase the Critical Heat 
Flux $^{9,11}$, that limits heat transport at the surface, or even to produce super-condensing devices. Controlling the wetting properties of these textured surfaces is thus a key ingredient for many applications. Another issue is the connection between the (static) wetting properties and the (dynamic) transport mechanisms (friction with the liquid in contact for example $)^{24}$. We shall consider in this article these two aspects for different types of structured surfaces, and show that metastability is an important mechanism to understand and design new surfaces with complex and interesting properties.

To introduce the problem, we shall first consider the example of superhygrophobicity, and generalize afterwards to other types of surfaces. Micro or nano-texturing an initially smooth surface increases its roughness, and thus amplifies its wetting properties since the contact area between the surface and the liquid is increased (Wenzel theory ${ }^{25}$ ). An initially hygrophobic smooth surface should become even more hygrophobic after structuring. A wetting transition can be induced by roughness as well when hygrophobicity becomes large - as shown by Cassie and Baxter ${ }^{1}$, leading to the "superhygrophobic" state. While the Wenzel state corresponds to a contact of the whole surface with the overlaying liquid, the Cassie-Baxter state corresponds to a composite wetting, where the liquid phase is suspended on the top of the asperities, with a gas phase (air or vapor or both) trapped in between (see Fig. 1). The Cassie-Baxter state is also referred to as the "Fakir" state, and we shall use this simple idiomatic terminology in the following. By opposition, the Wenzel state is often called the "impaled" state, and we shall again use this name in what follows. The Fakir state is interesting since the gas layer trapped between the surface and the liquid plays the role of a lubricant, and can induce a large slippage of the fluid ${ }^{13,15-18}$. Slippage is however not perfect since it competes with the friction applied by the roughness ${ }^{26,27}$. Efficiency of slippage is characterized by the slip length $b$, that gives the position inside the surface where the extrapolated velocity field cancels (see Fig. 2). It has indeed been shown by many authors ${ }^{13}$ that far away from any kind of surface, the velocity field corresponds to the field produced by an effective planar surface with the partial-slip - or Navier - boundary condition (BC):

$$
v_{\|}\left(z_{\mathrm{s}}\right)=\left.b \frac{\partial v_{\|}}{\partial z}\right|_{z=z_{\mathrm{s}}},
$$

relating the slip velocity $v_{\|}$, the slip length $b$ and the local shear rate on the effective planar surface - often named the 'plane of shear' - placed in $z=z_{\mathrm{s}}$ (see Fig. 2). The slip length is very sensitive to the details of the surface texture: Anisotropic textures usually leads to anisotropic slippage, giving rise to interesting applications (mixing in microchannels ${ }^{28}$ for instance).

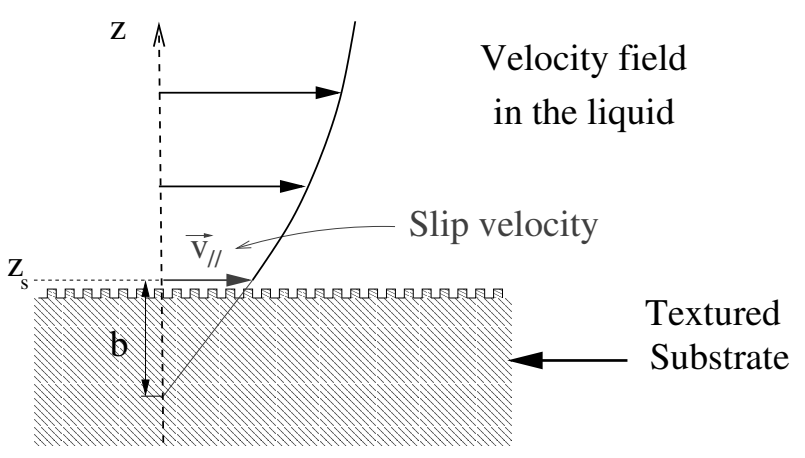

Figure 2: Partial slip boundary condition: definition of the slip length $b$ and of the 'shearplane' position $z_{\mathrm{s}}$.

More generally, the link between the wetting properties of the textured surface, and of an ideally smooth surface of the same chemical nature (the reference surface), is plotted on a wetting diagram. This diagram gives the variation of the effective contact angle $\theta_{\text {eff }}$ (more precisely $\left.\cos \theta_{\text {eff }}\right)$ of a liquid placed on the textured surface as a function of the intrinsic contact angle $\theta$ (in fact $\cos \theta$ ) of the reference smooth surface. The wetting states are clearly visible on these diagrams since they give rise to different branches; The transition points can be identified by the intersections of the branches. The wetting diagram is obtained by using the Young law:

$$
\cos \theta_{\mathrm{eff}}=\frac{\gamma_{\mathrm{SV}}^{\mathrm{eff}}-\gamma_{\mathrm{SL}}^{\mathrm{eff}}}{\gamma_{\mathrm{LV}}},
$$

where $\gamma_{\mathrm{LV}}$ is the liquid-gas surface tension, $\gamma_{\mathrm{SV}}^{\mathrm{eff}}$ is the effective surface tension between the gas 
and the substrate, and $\gamma_{\mathrm{SL}}^{\text {eff }}$ is the effective surface tension between the liquid and the substrate. By "effective" we mean that this surface tension is the global surface energy divided by the apparent macroscopic area of the surface (the asperities are not visible at the macroscopic scale, and the textured surface look smooth at this scale). For a particular geometry of the surface, and making assumptions on the possible wetting states, it is possible to get theoretical expressions for these effective surface tensions and to obtain the corresponding branches. We show in Fig. 3 the shape of this diagram for a crenelated surface, where we can identify three branches. While the first one (I) corresponds to the Cassie-Baxter state, a competition between a fakir state for the liquid, and an impaled state for the gas in contact with the surface (see Fig. 3), branch II corresponds to an impaled state for both the gas and the liquid phase (the situation considered by Wenzel), and branch III, very promising in the context of condensation and boiling, corresponds to an impaled state for the liquid, and to a composite wetting situation - with liquid condensed in the asperities - for the gas-substrate surface tension. We shall refer to this last state as the "inverse-fakir" state, since it is the exact inversion of the fakir state described above. As a generic feature, we can see that the structuring of a surface can only enhance its natural wetting property, a smooth hygrophilic surface will become more hygrophilic after texturing, and a smooth hygrophobic surface will become even more hygrophobic once textured. Reversing the wettability is not possible if we refer to Fig. 3.

This quite generic wetting diagram, obtained in the macroscopic framework introduced by Wenzel, Cassie and Baxter raises in fact many questions. First, it is derived from a purely macroscopic approach and we can wonder if it is still valid at the nanometric scale. This question received a positive answer thanks to the molecular simulations done by Cottin-Bizonne et al. ${ }^{29}$ : The Cassie-Baxter - Wenzel transition is indeed observed even for nanostructured surfaces. Next, only a limited number of wetting states are taken into account in this macroscopic approach, the lowest surface energy state being

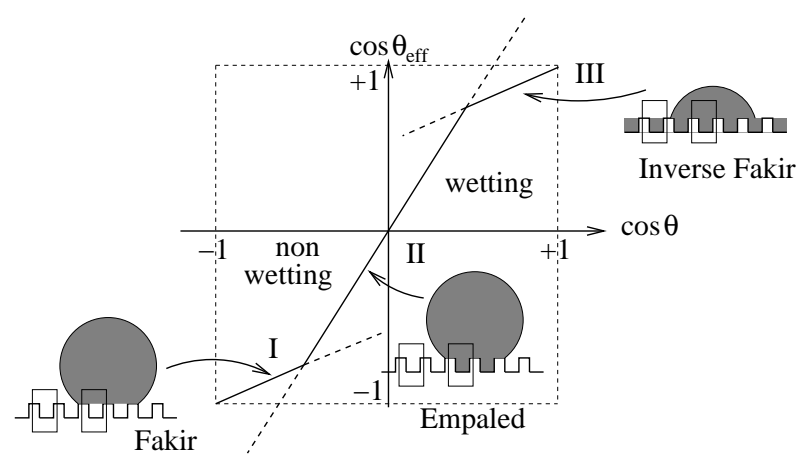

Figure 3: Wetting diagram for a crenelated surface. The small boxes show the wetting states considered for the calculation of $\gamma_{\mathrm{SV}}^{\mathrm{eff}}$ and $\gamma_{\mathrm{SL}}^{\mathrm{eff}}$.

considered as stable. We can thus also wonder if all the possible states have been found. A more systematic approach would consist in minimizing the free energy of a system presenting a liquid-gas coexistence in contact with the textured substrate. Such an analysis is presented in $^{12}$, with some restrictions (planar interfaces, macroscopic theory), leading to analytical results. We used a more general approach in a previous study ${ }^{30}$, based on numerical minimization of a free energy model, and showed that the Wenzel - Cassie - Baxter framework is correct even at very small scale, provided little corrections accounting for corner energies are made. But the most interesting result concerns metastability. The wetting transitions are first order phase transitions and metastable states are possible. Metastability has already been reported in many experimental situations ${ }^{31-33}$, and a very important question is to understand the mechanisms that control it ${ }^{3,4,34-36}$. Metastability usually only delays a transition, but we shall see that depending on the structure of the surface, it can even lead to a reversal of the wettability. A study of metastability requires a model able to cope with free-energy barriers, and this is the reason why we used our free energy model to investigate this problem. Since we are also interested in the determination of the effective hydrodynamic boundary conditions e.g. the slip length - even in the metastable states, the free energy model is combined to a hydrodynamic equation to get both the static and the dynamic properties. We shall restrict 
our modeling to finite element computations when the thermodynamic aspects are not important.

We discuss in section 2 the main ingredients of the model; A more detailed discussion and justification will be found in ${ }^{30}$. The results are presented in section 3 for the wetting diagram and the discussion on metastability in the context of superoleophobic surfaces. In section 4 we consider the friction properties of these surfaces and section 5 is devoted to our conclusions.

\section{$2 \quad$ Model and Parameters}

Several approaches can be used to model the physics of a fluid close to an interface. At the nanometric scale a fluid is made of molecules, and the standard theoretical and numerical tools at these scales are statistical mechanics and molecular dynamics. These very powerful tools provide interesting informations on the structuring of the fluid close to a smooth surface, and give access to the thermodynamic and the dynamic properties of the fluid at these very small scales. Although fully general in their principle, applying these methods to microstructured surfaces is very difficult due to the huge number of molecules one need to consider at the micron scale $\left(10^{9}-10^{12}\right)$. Alternatively, continuum models are known to be valid even at very small scales: they can be applied down to the molecular scales with special care. The thermodynamic properties (phase behavior, wetting properties, interfacial tensions...) have been widely investigated in the context of statistical mechanics at the nanometric scale, within the framework of Density Functional Theory (DFT). DFT generalizes the macroscopic thermodynamic potentials, like the Free energy, to very inhomogeneous situations including fluidsurface interactions or liquid-gas coexistences. Instead of minimizing a free energy, we need to minimize a free energy functional $F[\rho]$ where $\rho(\mathbf{r})$ is the density profile of the fluid close to the surface or at a liquid-gas interface. Although DFT provides a formal expression for this functional, it is far from explicit and approximate functionals must be used in practice. It is not our purpose to describe in details the molecular organization close to the surface; Our goal is rather to relate the effective macroscopic wetting and slip properties to the nanometric intrinsic properties of the interfaces. For the wetting properties, this connection is given by the wetting diagram where the macroscopic effective contact angle $\theta_{\text {eff }}$ is given as a function of the nanoscale intrinsic contact angle $\theta$ of the reference atomically smooth surface. To this end, we shall consider the simplest functional we can construct, including only the necessary ingredients to describe the wetting transitions: the free energy functional must account for a possible liquid-gas coexistence induced intrinsically by the roughness, and for the various surface tensions leading to the determination of the contact angles. The simplest free energy we can use to model a liquid-gas coexistence is given by the van der Waals equation of state; Inhomogeneities and interfaces can be treated in the simple square gradient approximation ${ }^{37}$ :

$$
F[\rho]=\int \mathrm{d} \mathbf{r}\left\{f(\rho)+\frac{w^{2}}{2}|\nabla \rho|^{2}+\rho V_{\text {wall }}(\mathbf{r})\right\},
$$

where $f(\rho)=k_{\mathrm{B}} T \rho\left\{\log \left(\frac{\rho b}{1-\rho b}\right)-1\right\}-a \rho^{2}$ is the van der Waals bulk free energy, the square gradient term $\frac{w^{2}}{2}|\nabla \rho|^{2}$ penalizes the formation of an interface and is thus responsible for the liquid-gas surface tension, and $V_{\text {wall }}(\mathbf{r})$ is the interaction potential between the molecules of the substrate and the molecules of the fluid. We use a Lennard-Jones potential to model these interactions: $V_{\mathrm{LJ}}(r)=4 \epsilon\left[(\sigma / r)^{12}-(\sigma / r)^{6}\right]$, where $\sigma$ is a molecular length. $V_{\text {wall }}(\mathbf{r})$ thus fixes the intrinsic wetting properties of the substrate, namely $\theta$ : by tuning the value of $\epsilon$ we can scan the whole range of wettability for the reference surface. A minimization of this functional can be performed in various ways, depending on the problem we wish to study. We shall introduce the Grand-potential functional $\Omega[\rho]=F[\rho]-\mu \int \mathrm{d} \mathbf{r} \rho(\mathbf{r})$ where $\mu$ is the chemical potential, to write the general equations used in this study. This thermodynamic approach will give us access to the wetting diagram, without a priori assumption on the possible wetting states, but it shall also provide informations on the metastability since the free energy barriers 
are included in the formulation.

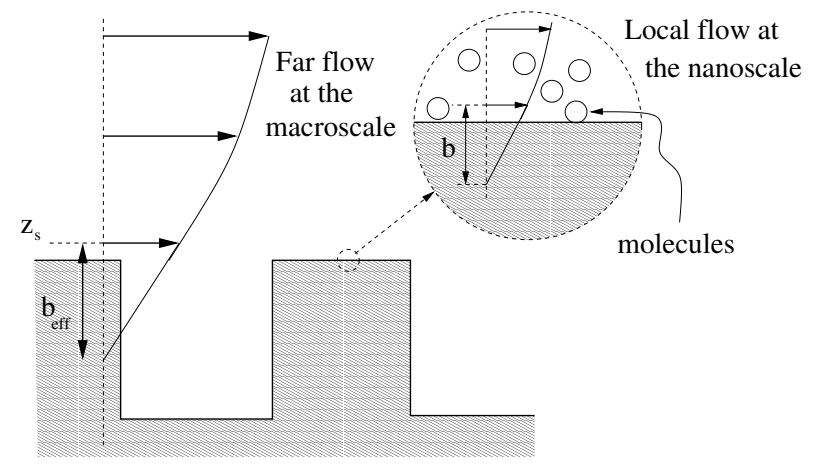

Figure 4: Definition of the slip lengths that enter into play for a patterned surface. $b$ is the nanometric (intrinsic) slip length, corresponding to the slip length on the reference smooth surface, while $b_{\text {eff }}$ is the effective slip length, induced by the structure.

In order to probe the dynamic of the fluid, we use a hydrodynamic transport equation to describe the velocity field close to the surface. The validity of hydrodynamics at very small scale has been checked both by molecular simulations $^{38,39}$ and by Surface Force Apparatus (SFA) experiments for simple molecular liquids like water or small alcanes ${ }^{40,41}$. The main concern with the use of hydrodynamic at the molecular scale comes from the boundary conditions (BC) that must be redefined. Molecular simulations and SFA experiments showed that the most general formulation for the hydrodynamic $\mathrm{BC}$ for a liquid close to an atomically smooth surface is the partial slip BC (1), where the slip length $b$ is a nanometric length in this case, and $z_{\mathrm{s}}$ is an atomic size (the effective planar surface is indeed located approximately one or two molecular layer inside the liquid for most liquids and atomically smooth surfaces ${ }^{38,39}$ ). Two very different slip lengths thus appear in the study of micro or nanostructured surfaces: the intrinsic slip length $b$ corresponding to the friction of the liquid on the surface at the molecular scale (see Fig.4), and the effective slip length $b_{\text {eff }}$ of the textured surface at the macroscopic scale. While $b$ results from the molecular interactions only ( $b$ is the slip length of the atomically smooth reference interface), $b_{\text {eff }}$ is due to the texture of the surface. $b$ remains of the order of $10 \mathrm{~nm}$ for usual non-wetting surfaces ${ }^{13}$, whereas $b_{\text {eff }}$ can reach the micron scale depending on the scale of the structure ${ }^{13}$. Since we are not interested in the nanometric effects controlling the intrinsic slip length $b$ in this study, we shall fix its value to reproduce the experimental and numerical observations, and focus our attention to $b_{\text {eff }}$ for different structures and wetting properties of the surface.

The hydrodynamic equation for the velocity field $\mathbf{v}$ reads:

$\frac{\partial \rho_{\mathrm{m}} \mathbf{v}}{\partial t}+\nabla \cdot\left(\rho_{\mathrm{m}} \mathbf{v} \mathbf{v}\right)=\nabla \cdot \sigma-\rho \nabla\left(\frac{\delta \Omega}{\delta \rho}\right)+\mathbf{f}_{\mathrm{wall}}$,

where $\rho_{\mathrm{m}}=M \rho$ is the local mass density ( $M$ is the molecular mass) and $\sigma=\eta(\rho)\left(\nabla \mathbf{v}+\nabla \mathbf{v}^{\mathrm{t}}\right)$ is the local viscous stress tensor $\left[\eta(\rho) \equiv \nu \rho_{\mathrm{m}}\right.$ is the local viscosity, with $\nu$ the kinematic viscosity, assumed to be constant in both phases]. The term exhibiting $\Omega$ in the hydrodynamic equation accounts both for the pressure tensor of the van der Waals fluid and for the capillary effects, as discussed above. To fix the intrinsic slip length $b$ of the reference surface we introduce an empirical friction force $\mathbf{f}_{\text {wall }}$ exerted by the walls on the fluid within a small range - equal to the range $\sigma$ of the wall/fluid interaction potential $V_{\text {wall }}(r)$, reflecting the friction force exerted by an atomically smooth wall on the first layer of fluid molecules.

$$
\mathbf{f}_{\mathrm{wall}}=-k \epsilon \rho(\mathbf{r}) \mathbf{v}(\mathbf{r}) \rho_{\mathrm{wall}}(\mathbf{r}) .
$$

This force is proportional to the interaction energy $\epsilon$ between the solid and the fluid ${ }^{13,39}$, and to the density of interacting molecules: the molecules of the fluid - through $\rho(\mathbf{r})$, and the molecules of the wall represented by the local density $\rho_{\text {wall }}(\mathbf{r})$. With the spontaneous depletion of the liquid from non-wetting walls, this enabled us to reproduce quantitatively the evolution of $b$ with $\theta$, as will be detailed in section 4 . $b$ varies from 0 for wetting situations $\left(\theta \sim 0^{\circ}\right)$ to $b \sim 10 \mathrm{~nm}$ (depending on the value of $k$ ) in nonwetting cases $\left(\theta \sim 120^{\circ}\right)$. To account for the phase equilibrium and the macroscopic transport, the minimization of the grand-potential is performed through an Allen-Cahn or CahnHilliard equation including the convective trans- 
port:

$$
\frac{\partial \rho}{\partial t}+\nabla \cdot(\rho \mathbf{v})=-C\left(\frac{\delta \Omega}{\delta \rho}\right) \quad \text { or }=C^{\prime} \Delta\left(\frac{\delta \Omega}{\delta \rho}\right)
$$

The first writing is a non-conserved Allen-Cahn dynamics, convenient for the study of phase equilibria since the system is in contact with a reservoir of matter fixing the chemical potential $\mu$; in that case $C$ is a constant fixing the relaxation time to equilibrium. The second writing corresponds to a more realistic conserved Cahn-Hilliard dynamics, where the number of molecules is held constant; The constant $C^{\prime}$ is now related to the self diffusion coefficient of the molecules in the fluid, and can be adjusted to match the proper value in the liquid under study. This Cahn-Hilliard formulation is useful, for instance, to study the friction properties of the liquid on the wall.

Once the intrinsic slip length $b$ and the intrinsic wetting angle $\theta$ of the reference atomically smooth surface have been fixed, the two equations (4) and (6) can be solved iteratively to measure the effective wetting angle $\theta_{\text {eff }}$ and slip length $b_{\text {eff }}$ of a textured surface. The texture is defined through the spatial variation of $\rho_{\text {wall }}(\mathbf{r})$, that can be chosen at will to represent crenelated surfaces, surfaces with posts or reentrant geometries as recently studied in the context of superoleophobic surfaces.

\section{$3 \quad$ Wetting properties}

Two different patterned surfaces are considered in this work, both with a ridged-type patterning, enabling us to solve the equations in two dimensions. First we consider a simple crenel geometry (Fig 5.a), with the following default parameters: $L=20 \mathrm{~nm}, W=2.5 \mathrm{~nm}$ and $H=10 \mathrm{~nm}$. This type of structure is often used to produce Super-Hydrophobic (SH) surfaces after a chemical coating with silane molecules. The second type of structure is a re-entrant Tshaped structure (Fig 5.b), with the following default parameters: $L=100 \mathrm{~nm}, W=20 \mathrm{~nm}$, $R=2.5 \mathrm{~nm}$ and $H=17.5 \mathrm{~nm}$. This type of structure has been considered to build SuperOleophobic (SO) surfaces, and we shall investi-
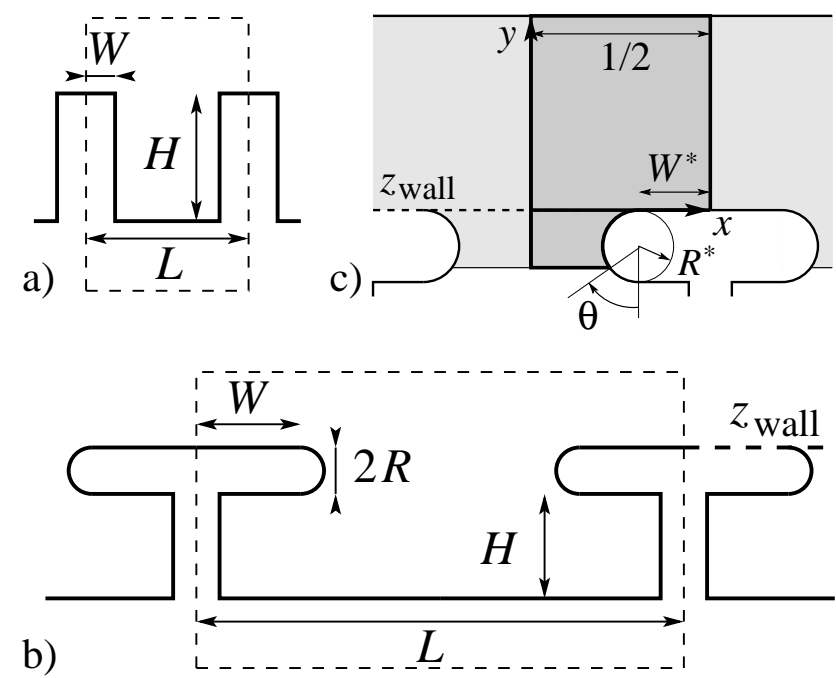

Figure 5: Systems under consideration. (a) crenelated surface; (b) re-entrant surface; (c) System considered for the finite element modeling, where $W^{*}=W / L$ and $R^{*}=R / L$.

gate the very different wetting behavior of these two types of surfaces in this part.

Two possible ways can be used to determine the contact angles. The most direct way is to measure the shape of a drop placed on the surface, and determine the angle it forms with the substrate at the triple line. The second way is to measure the various surface tensions and use the Young law (2) to deduce the wetting angle. While the first method is the experimentally efficient way to proceed, the second is in fact the most efficient strategy from the numerical point of view. Although a direct measurement of the contact angle of a drop on a smooth substrate is possible - and has been done as a check, this method is far from optimal since it requires a huge amount of computation time - due to the large scale of the drop, and a careful adjustment of the drop shape - a source of errors. For textured surfaces, a direct measurement of the contact angle is plagued by the roughness: Many metastable states can indeed exist at the triple line, forcing the drop size to be several orders of magnitude larger than the roughness amplitude to expect an accurate determination. This method is not tractable in practice. On the contrary, the measurement of the surface tensions only requires small scale computations (as pre- 
i)

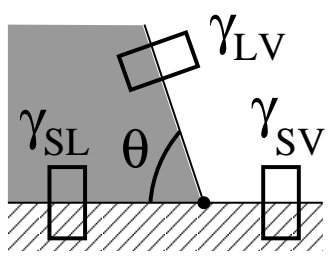

a)

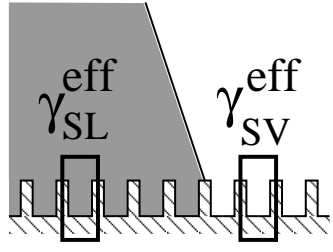

b)

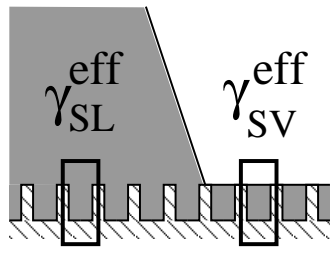

c)

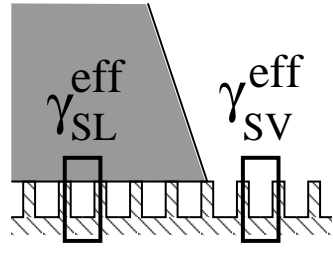

Figure 6: Contact angle measurement: zoom on the contact line region i) On a smooth surface (Young contact angle); a, b, c) On a patterned surface (effective contact angle) in various configurations: (a) impaled drop surrounded by a dry substrate, with both liquid and vapor in the impaled state; (b) impaled drop surrounded by a wet substrate, with the liquid in the impaled state and the vapor in the inverse-fakir state; (c) fakir drop surrounded by a dry substrate, with the liquid in the fakir state and the vapor in the impaled state

sented in Fig. 6 where the small rectangles represent the simulation boxes), a single pattern is sufficient to determine the various surface tensions, and this method is numerically very accurate since no adjustment needs to be done (the interfacial energy directly results from the freeenergy model we consider).

The wetting diagram for the two types of structured surfaces described above is probed using the strategy presented in the previous section: the intrinsic contact angle $\theta$ is tuned by changing the interaction potential between the molecules of the fluid and the molecules of the substrate (parameter $\epsilon$ ), and the resulting effective contact angle induced by the structure is determined by a measurement of the surface tensions. To measure $\gamma_{\mathrm{SV}}^{\mathrm{eff}}$ we start the computation with a gas phase in contact with the textured surface, and after equilibration the surface energy is computed from the free energy functional. An identical procedure is applied for

$\gamma_{\mathrm{SL}}^{\mathrm{eff}}$, where the liquid phase is placed in contact with the textured surface. $\gamma_{\mathrm{LV}}$ does not need to be measured (although we did it to test the accuracy of the numerical scheme) since there is an analytical expression for it ${ }^{37}$. To probe metastability, we can vary the initial configurations. Two types of initial configurations have been used for each simulation: An initial configuration corresponding to an impaled state (for example a texture initially filled with liquid for the determination of $\gamma_{\mathrm{SL}}^{\mathrm{eff}}$ ) and a composite wetting situation (Fakir state for $\gamma_{\mathrm{SL}}^{\mathrm{eff}}$ ). After equilibration, only one state should be obtained if there were no metastability.

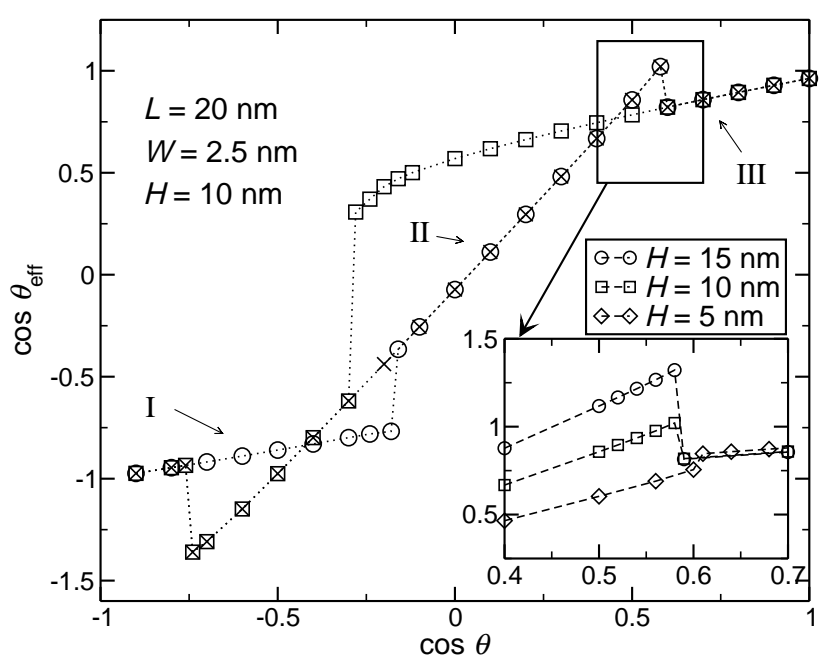

Figure 7: Wetting diagram of the crenelated surface, using various initial wetting configurations: (cross): both liquid and vapor in the impaled state (Fig 6.a); (square): liquid in the impaled state and vapor in the inverse-fakir state (Fig 6.b); (circle): liquid in the fakir state and vapor in the impaled state (Fig 6.c). Inset: Zoom on the filling transition for the vapor/solid interface, with both liq./sol. and vap./sol. interfaces initially in the impaled state (Fig 6.a), and for various crenel heights.

The results are plotted in Fig. 7 for the crenelated surface, as reported in a previous article $^{30}$. This figure illustrates different features of the wetting transitions. First the overall scenario obtained within the macroscopic CassieBaxter - Wenzel approach (Fig. 3) is well reproduced, the three branches corresponding to the stable wetting states are easily observed. We 
also observe an asymmetry of the wetting diagram not predicted by the macroscopic model. This asymmetry between the wetting and the non-wetting part of the diagram is due to local effects at the internal corners of the structure: an atom of the fluid suffers more attraction from the wall in this region than anywhere else, and the liquid phase is thus favored at the inner corners. While the consequences of these corner effects on the wetting diagram have been discussed in ${ }^{30}$, we mention here that they only modify the position of the wetting branches in a moderate way; The precise effect depends on the size of the texture and becomes negligible for large scale (micronic) textures. But we shall see in the following that the corners actually control the metastability, and this effect remains important even for large scale textures. Finally, we can note that the three branches give rise to metastable states, and we shall investigate below the mechanisms that control this metastability. Before discussing this important point, we must note that an effective non-wetting behavior can only be obtained when the surface is intrinsically (i.e. chemically) non-wetting: the lower right quadrant corresponding to intrinsic wetting and effective non-wetting is not reached. From this point of view, a surface that is intrinsically oleophilic (a common situation for superhydrophobic surfaces) cannot be converted to a superoleophobic surface with this kind of structure. These crenelated surfaces are thus not good candidates to repel both water and oils.

If we now consider the re-entrant geometry schematically represented in Fig 5.b, the same study can be done, and the corresponding wetting diagram is plotted in Fig 8. We can observe on this diagram the strong enhancement of the metastability, in particular, the fakir branch (I) now reaches the lower right quadrant, indicating the possibility for the surface to be superhygrophobic while the overlaying liquid is intrinsically hygrophilic.

This situation is however metastable: one can observe in Fig 8 that above $\cos \theta \simeq 0.58$, the fakir state I (Fig 6.c) collapses to the inversefakir stable branch III (Fig 6.b). The wetting properties of the surface are thus abruptly

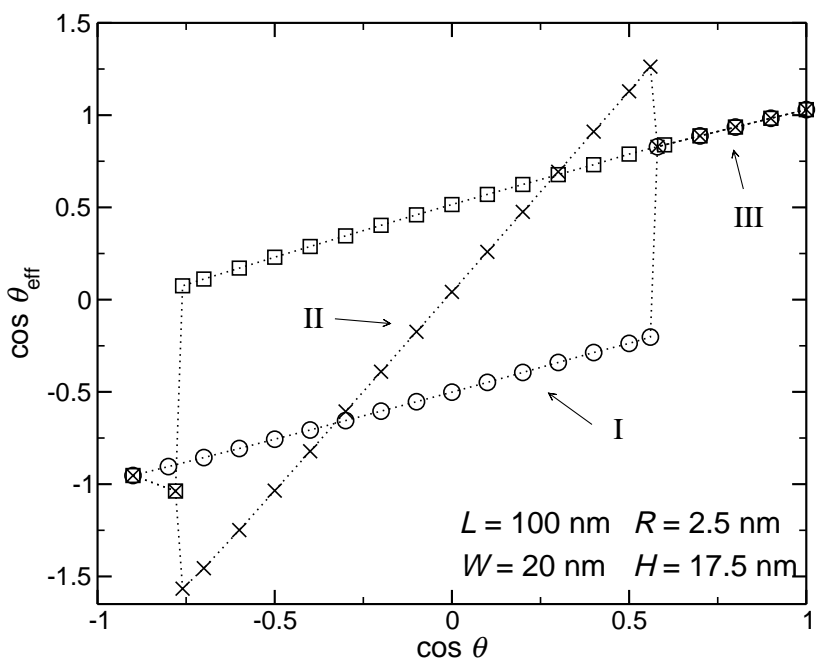

Figure 8: Wetting diagram of the re-entrant surface, using the same notations than in Fig 7.

reversed at this point. This loss of superrepellency is due to the filling transition: the cavity initially filled with gas in the fakir state suddenly fills with the liquid phase. A control of the factors entering into this transition should enable us to improve those surface, in particular to increase the metastability. Let us thus now

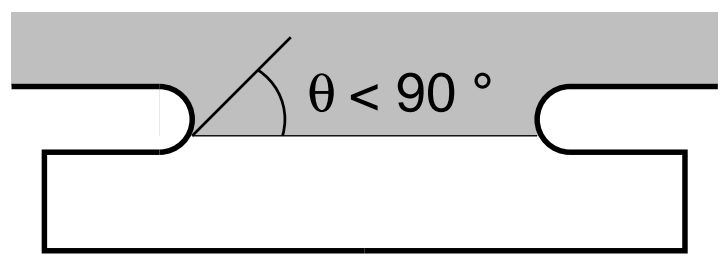

Figure 9: Basic principle of superoleophobicity: the pinning of the meniscus at the re-entrant geometry ensures the metastability of the fakir state even for a intrinsically wetting surface.

turn our attention to the filling transition itself. Since the intrinsic contact angle $\theta$ is finite, the meniscus is pinned onto the perfectly re-entrant surface (Fig 9), and cannot penetrate inside the cavity. Therefore, this filling transition must rather originate in the spontaneous filling of the cavities with the liquid phase rather than in the collapse of the meniscus. Several features observed in Fig 8 are supporting this idea: The filling transition connects the two branches I and III, which means that the transition affects both 
the liquid/solid and the vapor/solid interfaces; Since the filling of the cavities with the liquid phase does not depend on the nature of the top phase, it is thus a property of the cavity itself. We can also observe that this transition occurs at the same value of the intrinsic contact angle, $\cos \theta \simeq 0.58$, for both the re-entrant surface (Fig 8) and the crenelated surfaces - whatever the height of the crenels (see the inset of Fig 7). This insensitivity of the transition to the global shape of the cavity enables us to conclude further that the mechanism responsible for the filling of the cavities with the liquid is a local effect. In fact we can monitor the transition with our dynamical model and see that the filling of the cavities with the liquid phase is due to a local condensation of the liquid at the internal corners of the cavity. A movie of the transition for re-entrant surfaces is available as an Electronic Supplementary Information (ESI), and an isodensity plot is given in Fig. 11 for a crenelated surface. The internal corners of the structure are very favorable sites for the molecules of the fluid since they feel at this particular place the attraction of the two faces of the corner. This excess of attraction at the internal corners confers to these sites the property to be natural nucleation sites: liquid droplets spontaneously form at the corners and progressively fill the cavity. To understand why the liquid forms spontaneously at the corners, we can follow the density of the gas phase at these points when we approach the transition value $\cos \theta \simeq 0.58$. This density in fact corresponds to the maximum density of the gas phase in the cavity. A plot of this quantity for a crenelated surface is given in Fig 10 as a function of the intrinsic contact angle $\theta$. When the surface gets more wetting, the strength of the fluid/wall interaction increases, and the peak density as well. When it reaches a value close to $\rho_{\max } \sigma^{3}=0.45$, the liquid fills the crenel and the peak density becomes that of the liquid. We can understand this behavior if we plot the bulk Grand-potential of the fluid as a function of the density, as shown in the inset of Fig 10. The two minima correspond to the bulk vapor and liquid densities, and a barrier stands between them. When the corner density crosses the Grand-potential barrier we can expect the

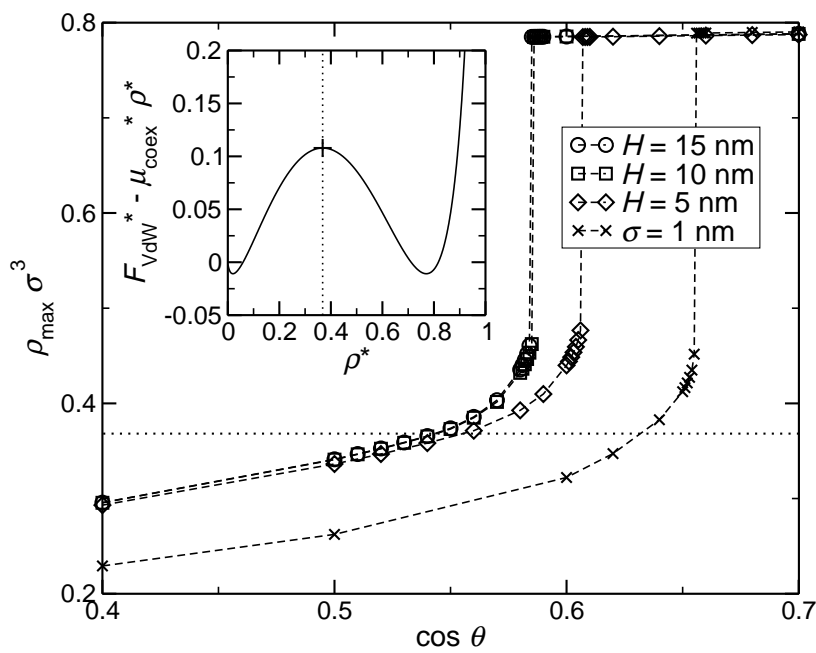

Figure 10: Formation of liquid drops at the inner corner of a crenelated surface: Evolution of the maximum fluid density at equilibrium inside the cavity with $\cos \theta$, for different crenel height $H$ and varying wall/fluid interaction range $\sigma$; When not specified, $H=10 \mathrm{~nm}$ and $\sigma=0.75 \mathrm{~nm}$. Inset: Representation of the adimensional Grand-potential governing the fluid behavior as a function of the numerical density $\rho$ of the fluid.

liquid phase to form spontaneously at the corner, but this transition is only local and an extra energy contribution must be added to form the liquid-vapor interface. It is thus not a surprise if the critical value of the density is significantly higher than the value corresponding to the maximum of the Grand-potential. We mention that the value $H=5 \mathrm{~nm}$ is a bit particular since the crenels in this case are so small that we cannot truly distinguish the fakir state from the impaled state. With this interpretation, the transition should be a first order transition involving nucleation-growth processes. In the framework of our model, we do not account for the thermal fluctuations and thus cannot study the nucleation process itself. But we can capture spinodal decompositions, and the liquid formation originates in an instability reminiscent of the crossing of a spinodal line in our approach. To emphasize this point, we plotted in Fig 11 the variation of the average Grand-potential as a function of time. The latter decreases slowly at the beginning, and then faster until the two 
droplets formed at each corner enter in contact and merge. The initial configuration is thus unstable, which corresponds to a spinodal situation. We expect that in experimental systems, thermally activated nucleation will be the predominant mechanism, and the transition should thus occur slightly below the critical value we measured in this study.

Now that the mechanism underlying the filling of the crenel has been identified, we understand that sharp corners inside the structure must be avoided if one wants to extend the metastability toward more wetting substrates. We applied this principle to our system by smoothing the fluid/wall interaction potential with a gaussian of width $0.5 \mathrm{~nm}$. For a given intrinsic contact angle, the peak density at the inside corners is then much lower, and no filling of the crenels could be observed until $\cos \theta \rightarrow 1$. We also used a slightly larger wall-fluid interaction range $\sigma$, here again diminishing the peak density at the corners and therefore extending the metastability toward more wetting situations (Fig 10).

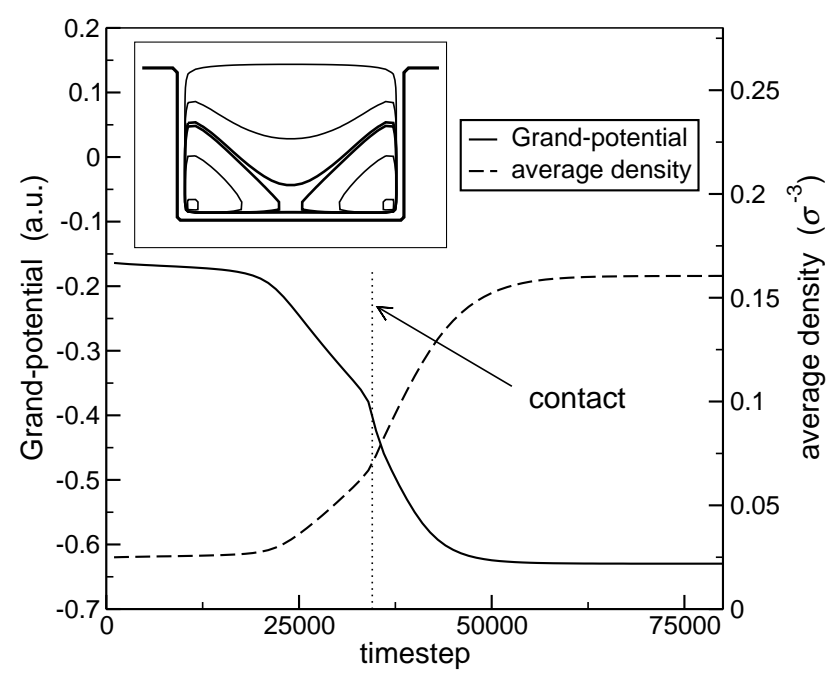

Figure 11: Filling transition on a crenelated surface: Evolution of the total Grand-potential and average density of the fluid in the system during the filling of the crenel with the liquid phase. Inset: snapshots of isodensity $\left(\rho \sigma^{3}=0.4\right)$ lines after 0, 27000, 34000 (just before the contact of the two drops), 35000 (just after the contact), 39000 and 80000 time steps.

\section{Friction properties}

We have shown in the previous section that re-entrant geometries can provide metastable superhygrophobic fakir states subsisting even when the surface is intrinsically hygrophilic. We are now going to investigate the possibility to obtain very low frictions (or equivalently very large effective slip lengths) of liquids on these surfaces by using this fakir state. To measure the effective slip length $b_{\text {eff }}$ of the reentrant surfaces, we confined a liquid between a smooth wall (top wall) and the structured surface (lower wall), and probed the effective hydrodynamic Boundary Conditions (BC) on each wall (Fig 12). This geometry enables us to get both the intrinsic slip length $b$, that corresponds to the atomically smooth top wall, and the effective slip length $b_{\text {eff }}$ measured for the structured lower wall, in the same conditions. It must be noted that the partial-slip BC depends on two parameters, the slip length, but also the position $z_{\mathrm{s}}$ where the boundary condition is applied, sometimes referred to as the 'plane of shear'.

For smooth surfaces, as already discussed in section $2, z_{\mathrm{s}}$ is in general a nanometric distance from the wall, but for structured or rough surfaces, this parameter will give the effective hydrodynamic position of the surface. Let us thus use the notation $z_{\mathrm{s}}^{\text {eff }}$ to identify the position of this plane for a textured surface; $z_{s}$ will be reserved for the reference smooth surface.

The determination of the two parameters $b_{\text {eff }}$ and $z_{\mathrm{s}}^{\text {eff }}$ requires the knowledge of the system response to two different types of flow. We will therefore use a Couette and a Poiseuille flow for this identification. A good indicator of the slip amplitude, often considered experimentally, is the extrapolated no-slip plane position $z_{\text {no-slip }}^{\text {eff }}=z_{\mathrm{s}}^{\text {eff }}-b_{\text {eff }}($ Fig 12): the deeper is this position inside the solid, the better is the liquid transport through the channel. We plotted in Fig 13 its evolution for the smooth and textured surfaces, as a function of the intrinsic contact angle $\theta$. On the smooth surface, our model - including a friction force exerted by the walls on the fluid within a small range, as detailed in section 2 - reproduces quantitatively the expected behavior, with almost a no-slip $\mathrm{BC}$ in the 


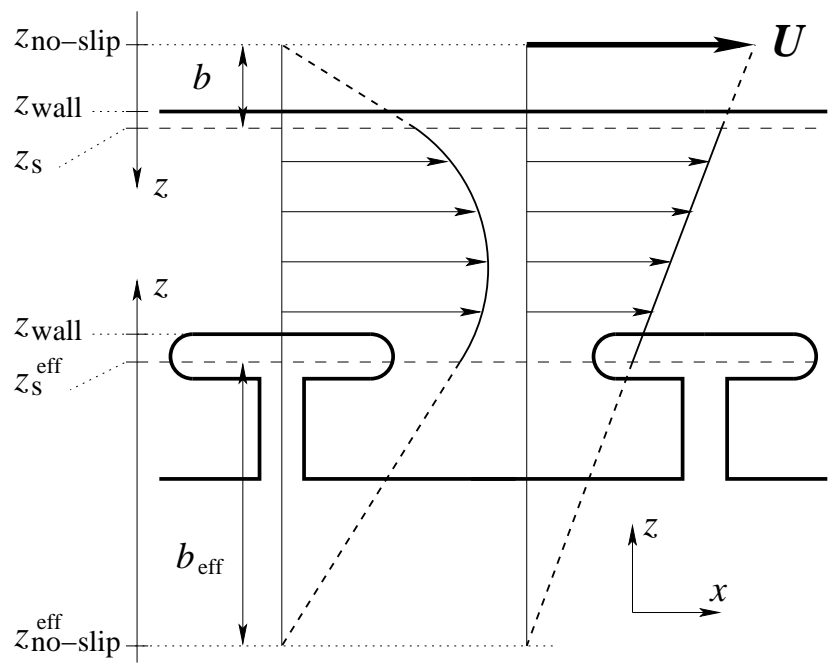

Figure 12: System used to probe the friction properties on re-entrant surfaces: A liquid is confined between a smooth wall and the ridged surface presented in Fig 5.b. In order to determine completely the effective hydrodynamic $\mathrm{BC}$ (i.e. measure both the shear plane position and the slip length) on each confining wall, a Couette and a Poiseuille flow perpendicular to the ridges are imposed to the confined liquid, and the (x-averaged) velocity profiles along $z$ are fitted in the middle of the channel, away from the surfaces. It is then straightforward to compute the effective no-slip plane position $z_{\text {no-slip }}^{\text {eff }}=z_{\mathrm{s}}^{\text {eff }}-b_{\text {eff }}$.

hygrophilic limit, and a few tens of nanometers slip length for highly hygrophobic surfaces.

More interesting is the slippage on the reentrant ridged surface. A striking point is the quick decrease of liquid slippage as the surface becomes more wetting, even though the surface remains in the fakir state; besides, we did not observe a large change of the slip behavior before and after the filling transition. This can be understood by looking at the dissipation around the structure, as detailed in the ESI. We observed that the maximum value of the viscous dissipation comes from the curved sections of the surface that are oriented at $45^{\circ}$ from the shear direction, due to the elongational component of the flow. A consequence of this large viscous dissipation at the upper edges of the structure is the dramatic reduction of the slip

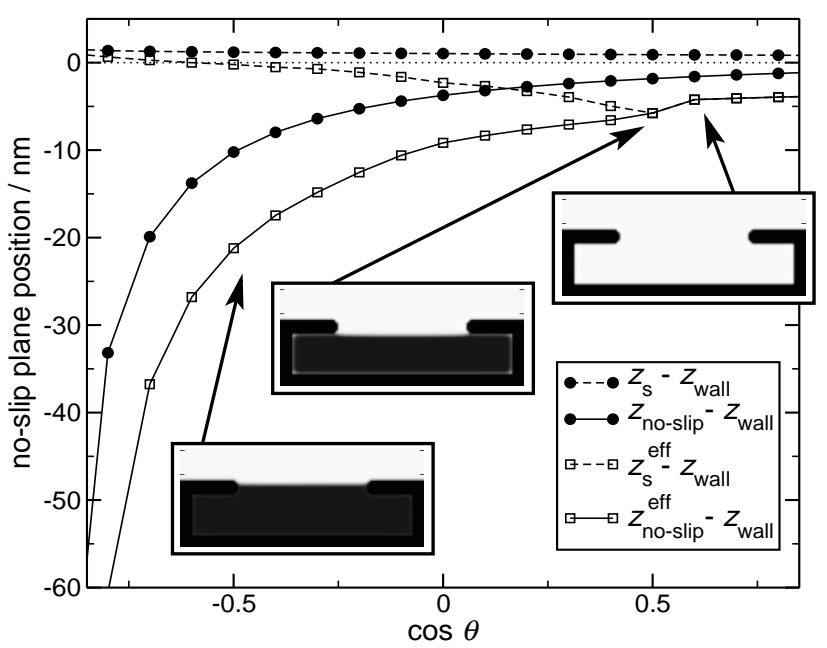

Figure 13: Evolution of the extrapolated no-slip plane position as a function of the Young contact angle $\theta$, for a smooth wall and a re-entrant ridged surface; Notations are explained in Fig 12 and its caption; For the structured surface, $z_{\text {wall }}$ is chosen to be the top of the pattern (see Fig 12). The insets represent three fluid density maps at the SO surface, for $\cos \theta=-0.5$; 0.5 and 0.6 .

length once the fluid starts to penetrate in between the plates. When $\cos \theta=0.5$, one can see in Fig 13 that there is no significant slippage left, although the cavity is filled with gas. The filling of the cavity with the liquid phase, as observed for $\cos \theta=0.6$, does not change much the situation and only very small differences can be observed in the dissipation profiles (see the ESI), with very little consequences on the slip properties. It has been known since the work of Richardson ${ }^{26}$ that roughness goes against hydrodynamic slippage, possibly leading to an effective no-slip BC on a locally perfectly slipping surface. Recently, Steinberger et $a l .{ }^{27}$ have shown that in the presence of a curved meniscus, SH surfaces could even slip less in the fakir state than in the impaled one. Our point here is that for a re-entrant surface in the fakir state, the curvature of the solid surface reduces slippage even in the ideal situation of a perfectly flat liquid/vapor meniscus.

In order to quantify the influence of geometry and contact angle on the slip properties of such surfaces at reasonable computational cost, we 
turned to finite element calculations. We modeled a newtonian liquid in the Stokes regime confined between a smooth wall and the reentrant ridged surface, in the fakir state, as illustrated in Fig 5.c. The results are detailed in ESI. On the whole, one can note that the slip properties can be affected significantly for wetting liquids, as soon as the radius $R$ of the 'reentrant' structure (see Fig 5 for the definition of $R$ ) is larger than typically one hundredth of the pattern periodicity $L$. It is therefore crucial to use an extremely sharp 're-entrant' structure (with $R / L<0.01$ ) if one wants oil to slip efficiently on such surfaces.

\section{Conclusion}

In this article, we have shown that our recently developed free-energy approach could account for the exceptional wetting properties of the reentrant textured surfaces used for the production of superoleophobic surfaces. In particular, we investigated the mechanism underlying the "filling" transition for the liquid on a such a surface. The liquid/vapor meniscus being unable to invade the re-entrant geometry, this transition originates in the spontaneous filling of the inner cavity with liquid, when the solid surface becomes locally too wetting. We have shown that the filling process was favored by the presence of sharp corners inside the structure; Consequently, internal corners must be avoided in the design of SO surfaces in order to optimize their wetting properties. From the point of view of the slip properties of liquids on SO surfaces, we have shown that the re-entrant curvature in the vicinity of the contact line introduces an additional friction and decreases hydrodynamic slippage. Consequently, SO surfaces should be designed with an extremely flat top and the sharpest 're-entrant' geometry as possible, in order to provide the best slip properties.

\section{Acknowledgments}

We would like to thank H. Rathgen for an enlightening discussion on SO surfaces, and gratefully acknowledge the suggestions of C. Cottin-
Bizonne and C. Ybert which helped us improve the manuscript. 


\section{References}

[1] A. B. D. Cassie and S. Baxter, Transactions Of The Faraday Society, 1944, 40, 0546-0550.

[2] C. Huh and S. G. Mason, J. Colloid Interface Sci., 1977, 60, 11-38.

[3] A. Marmur, Langmuir, 2003, 19, 83438348.

[4] N. A. Patankar, Langmuir, 2004, 20, 70977102.

[5] M. Callies and D. Quere, Soft Matter, 2005, 1, 55-61.

[6] M. L. Ma and R. M. Hill, Current Opinion In Colloid $\&$ Interface Science, 2006, 11, 193-202.

[7] P. Roach, N. J. Shirtcliffe and M. I. Newton, Soft Matter, 2008, 4, 224-240.

[8] S. Vemuri and K. J. Kim, International Communications In Heat And Mass Transfer, 2005, 32, 27-31.

[9] H. Kim, J. Kim and M. H. Kim, International Journal Of Heat And Mass Transfer, 2006, 49, 5070-5074.

[10] S. J. Kim, I. C. Bang, J. Buongiorno and L. W. Hu, Appl. Phys. Lett., 2006, 89, 153107.

[11] H. D. Kim and M. H. Kim, Appl. Phys. Lett., 2007, 91, 014104.

[12] A. Marmur, Langmuir, 2008, 24, 75737579 .

[13] L. Bocquet and J. L. Barrat, Soft Matter, 2007, 3, 685-693.

[14] H. A. Stone, A. D. Stroock and A. Ajdari, Annual Review Of Fluid Mechanics, 2004, 36, 381-411.

[15] J. Ou, B. Perot and J. P. Rothstein, Phys. Fluids, 2004, 16, 4635-4643.
[16] C. H. Choi and C. J. Kim, Phys. Rev. Lett., 2006, 96, 066001.

[17] P. Joseph, C. Cottin-Bizonne, J. M. Benoit, C. Ybert, C. Journet, P. Tabeling and L. Bocquet, Phys. Rev. Lett., 2006, 97, 156104.

[18] C. Ybert, C. Barentin, C. Cottin-Bizonne, P. Joseph and L. Bocquet, Phys. Fluids, 2007, 19, 123601.

[19] S. Herminghaus, Europhys. Lett., 2000, 52, 165-170.

[20] A. Tuteja, W. Choi, M. L. Ma, J. M. Mabry, S. A. Mazzella, G. C. Rutledge, G. H. McKinley and R. E. Cohen, Science, 2007, 318, 1618-1622.

[21] A. Ahuja, J. A. Taylor, V. Lifton, A. A. Sidorenko, T. R. Salamon, E. J. Lobaton, P. Kolodner and T. N. Krupenkin, Langmuir, 2008, 24, 9-14.

[22] L. L. Cao, T. P. Price, M. Weiss and D. Gao, Langmuir, 2008, 24, 1640-1643.

[23] J. Zimmermann, M. Rabe, G. R. J. Artus and S. Seeger, Soft Matter, 2008, 4, 450452.

[24] D. M. Huang, C. Sendner, D. Horinek, R. R. Netz and L. Bocquet, Phys. Rev. Lett., 2008, 101, 226101.

[25] R. N. Wenzel, Industrial And Engineering Chemistry, 1936, 28, 988-994.

[26] S. Richardson, J. Fluid Mech., 1973, 59, 707-719.

[27] A. Steinberger, C. Cottin-Bizonne, P. Kleimann and E. Charlaix, Nature Materials, 2007, 6, 665-668.

[28] A. D. Stroock, S. K. W. Dertinger, A. Ajdari, I. Mezic, H. A. Stone and G. M. Whitesides, Science, 2002, 295, 647-651.

[29] C. Cottin-Bizonne, J. L. Barrat, L. Bocquet and E. Charlaix, Nat. Mater., 2003, 2, 237-240. 
[30] T. Biben and L. Joly, Phys. Rev. Lett., 2008, 100, 186103.

[31] S. Moulinet and D. Bartolo, European Physical Journal E, 2007, 24, 251-260.

[32] M. Reyssat, J. M. Yeomans and D. Quere, Epl, 2008, 81, 26006.

[33] X. M. Li, T. He, M. Crego-Calama and D. N. Reinhoudt, Langmuir, 2008, 24, 8008-8012.

[34] C. Ishino, K. Okumura and D. Quere, Europhysics Letters, 2004, 68, 419-425.

[35] A. Dupuis and J. Yeomans, Langmuir, 2005, 21, 2624-2629.

[36] H. Kusumaatmaja, M. L. Blow, A. Dupuis and J. M. Yeomans, Epl, 2008, 81, 36003.

[37] J. S. Rowlinson and B. Widom, Molecular Theory of Capillarity, Oxford University Press, 1989.

[38] L. Bocquet and J. L. Barrat, Physical Review E, 1994, 49, 3079-3092.

[39] J. L. Barrat and L. Bocquet, Faraday Discuss., 1999, 112, 119-127.

[40] D. Y. C. Chan and R. G. Horn, J. Chem. Phys., 1985, 83, 5311-5324.

[41] C. Cottin-Bizonne, A. Steinberger, B. Cross, O. Raccurt and E. Charlaix, Langmuir, 2008, 24, 1165-1172. 